\title{
Networking Multiword Units
}

\author{
Matthieu Constant ${ }^{1}$ and Patrick Watrin ${ }^{2}$ \\ 1 Université Paris-Est, IGM \& CNRS \\ ${ }^{2}$ Université catholique de Louvain, CENTAL
}

\begin{abstract}
This paper details a network infrastructure for representing and sharing multiword units. It enables connecting local networks describing linguistic semi-fixed components in the form of local grammars.
\end{abstract}

\section{Introduction}

Multiword units (MWUs) have deep impact on Natural Language Processing (NLP) because their lexical, syntactic or semantic behaviour is often unpredictable from their individual words [14]. In the past twenty years, researches pointed out the lack of data resources of these expressions and attempted to build broad-coverage lexicons, such as $[7,5,6]$.

Local grammars (LGs) [8] have been shown of great interest to represent multiword units and especially those involving some degree of lexical and syntactic variability [16, $13,4]$. Their easy integration in NLP applications is also very convenient like in $[9,15$, 3]. They are in the form of Recursive Transition Networks (RTNs) that describe word sequences in a factorized and structured manner. They form linguistic components that can be reused by other local grammars. Nevertheless, in pratice, local grammars are often stored on isolated machines and their components cannot be directly used by others. Our objective is to connect these local networks in order to facilitate collaborative work, to share them freely with the community and use them in NLP applications.

This paper describes an infrastructure for networking LGs and details different implemented services facilitating importation, access and publishing. This network is formed of three layers: (1) a formal network (RTN) representing word sequences; (2) a linguistic network combining linguistic components in the form of local networks; (3) a collaborative network connecting linguistic components of different authors. First, we describe local grammars as linguistic recursive networks and illustrate it with MWUs ${ }^{3}$. Next, we detail a colaborative infrastructure connecting local grammars together and the different services implemented. We finally present the current state of the system, which contains local grammars compatible with platforms Unitex [10] and Outilex [2].

\section{Local grammars as linguistic recursive networks}

The MWUs we are dealing with are contiguous semi-fixed collocations ${ }^{4}$ of two or more words, that can be considered as lexical units. There exist many varieties such as

\footnotetext{
${ }^{3}$ LGs can formalize other types of linguistic units (e.g. chunks or phrases). We decided to focus on MWUs because they are considered key elementary units in many modern applications.

${ }^{4}$ The continuity of the collocation is not strict because it might contain adjectival or adverbial inserts like in ministre [français] de l'Agriculture ([French] minister of Agriculture).
} 
nominal collocations (e.g. red wine), light verb constructions (to have the impression), compounds (e.g. of course, close to), auxiliary predicates (e.g. continue to $\langle$ verb $>$ ), named entities such as time adverbials (January 12, 2007) or person names (George W. Bush), and so on. Some of them accept lexical variations. For instance, in the expression (gold+silver+bronze) medal, the modifier paradigm is limited to a small semantic class. When lexical variation is limited to classes of few elements, this type of expressions might be listed in dictionaries. Nevertheless, some expressions accept larger variations that are not suitable to be listed in the form of dictionaries. For instance, the expression world champion may vary in $(E+$ defending + reigning) (European + African...+$)$ champion. In that case, it is more convenient to describe the variation with a more compact formalism like local grammars.

A local grammar [8] is a recursive transition network [17]. It is composed of recursive automata which transition labels can be either lexical items or calls to other automata. There exist a main automaton which is the entry point of the network. These grammars theoretically recognize algebraic languages. Numerical expressions are a typical kind of expressions the description of which local grammars is best suited for. A significant interest is that linguistic components described by local grammars can be used by other local grammars by means of a simple reference. For instance, the compound locative preposition around ten meters east of is recognized by the simple local grammar given in figure 1 . This grammar combines linguistic components defined

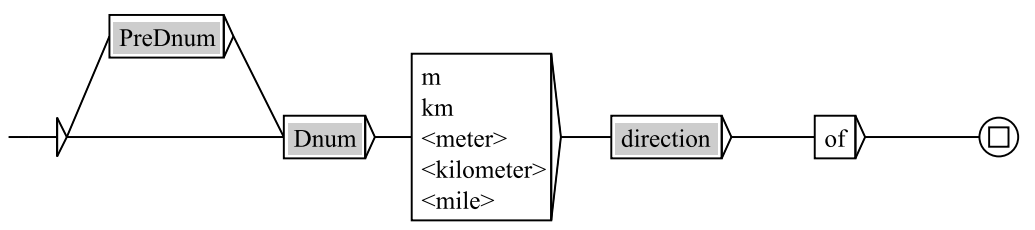

Fig. 1. Locative preposition

in other automata. PredDnum is an automaton that describes all possible numerical predeterminers such as around, approximatively. Dnum points out the automaton representing numbers. Direction is an automaton that recognizes different directions such as west, north, southwest. This grammar can be integrated to a larger grammar of compound prepositions by a simple reference to its axiom automaton.

\section{A collaborative network insfrastructure}

\subsection{A decentralized architecture}

In theory, a LG can use components of other LGs. Nevertheless, in pratice, authors do not have an overview of available components and they cannot directly use them in their own grammars because they are stored on isolated machines. We propose a decentralized network infrastructure to handle this issue. It consists of a set of HTTP servers (see figure 2). These servers are only used as repositories of LGs, that are managed independently of each others by their respective owners. Reporitories are in the most part 
located on the own web site of the grammar authors. A user that needs to get access to the LGs, uses an access server including an index. The queries are processed with the use of this index that gathers all data information of the network. The decentralized architecture is then invisible for the user.

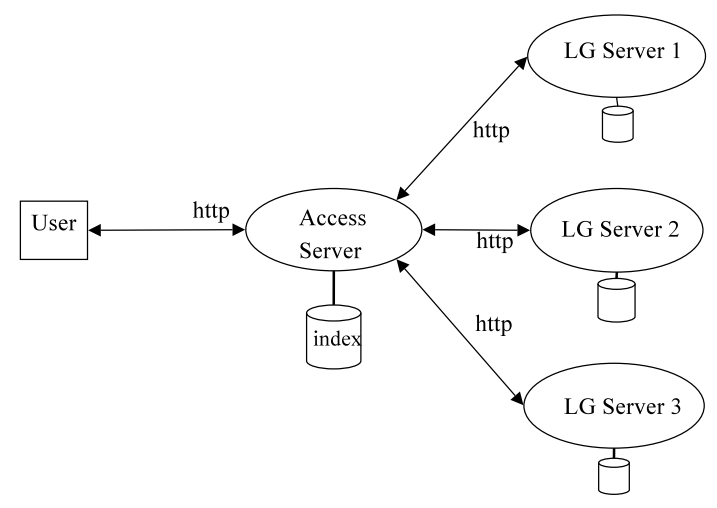

Fig. 2. Architecture

Each repository includes a collection of packages of local grammars. A package is an archive including a set of (recursive) automata, a license and documentations in XML and HTML. This documentation contains information such as authors, language, linguistic description, entry points (main automata), required preprocessing and linguistic resources (e.g. dictionaries). An automaton of a given package can call any automaton contained in a package referenced by the system. A system of automata referencing has been developped for that purpose.

The index contains precise information on repository packages, especially on the linguistic content. In its current state, it contains the following information: addresses of all repositories; packages (language, repository, path); terms used in the documentations and in the automata; dependency between the automata (which automata call which automata); entry points of the network (main automata).

\subsection{Web Services}

As any network containing data, this infrastucture requires some services to make it useful. In addition of a manager that helps publishing packages and importing grammars on local machines, we implemented a network browser and a search engine gathered in GraalWeb, a Java Applet ${ }^{5}$.

The search engine has been implemented to help users to find grammars. The query is a set of terms occurring either in the automata or in the documentation. The computation of the query produces a list of automata sorted according to their relevancy to the query. This tool is based on standard Information Retrieval techniques [1] by using space vector models for representing queries, automata and documentations. We developed three techniques to search grammars according to their lexical content. The first

\footnotetext{
${ }^{5}$ http://igm.univ-mlv.fr/ mconstan/library
} 
one consists in considering that the terms of an automaton are its lexical items. The similarity rate between an automaton and a query is the cosine between their respective vectors. The second technique (independent from the query) consists in computing a popularity rate for each automaton by using the PageRank technique used for Google search engine [11]: the more an automaton is called by popular automata, the more it is popular. We called this procedure GrammarRank as a tribute to its well-known inspiration. The third technique consists in combining lexicon and dependency. It is based on the fact that a term used in an automaton is also indirectly used by an automaton calling it. Our algorithm propagates terms in the inverted dependency graph of the library. Recent similar experiments have been conducted successfully for information retrieval on the web like in [12]. The final score of a retrieved automaton combines the three techniques the scores of which are assigned coefficients. When searching in the documentation content of the packages, we use a variant of the first technique. If a package is relevant, solely the main automata are listed.

The implemented browser provides global and detailed views of the network. Firstly, all available packages are listed and their documentation can be seen to get an overview of the linguistic content. The structure of the dependency between the automata of a package can be visualized in the form of a tree like in file system browsers. Each automaton can be explored in detail with a graph viewer implemented from Unitex source code. A call to another automaton is considered as an hypertext link that can be followed to be vizualized (by a simple mouse click). The browser is also used to follow the inverted dependency of the library, i.e. to get the list of automata that calls the current automaton, select one and vizualize it.

The manager tool especially provides a functionality that projects a local grammar of a package (including external components) on the filesytem of a local machine by keeping the structure of the network.

\section{Current State}

The networked library currently contains local grammars compatible with formats and resources of the Unitex and Outilex platforms. It includes 6 repositories and 9 packages. In total, 1,496 automata including 11 main automata, are referenced by the system. The network has 33,577 states and 77,978 transitions. For instance, we consider that the automaton in figure 1 has 7 states and 7 transitions. The dependency graph is composed of 5,258 edges. Two languages are represented: English and French. Several types of MWU grammars are referenced: sequences of determiners in French, e.g. la plupart des dix (most of the ten), named entities (location, organizations, persons, time) in French and English, sequences of verbs in French and English (has continued to be afraid of eating), locative prepositions in French (ten meters west of).

\section{Conclusion and Future Work}

The network infrastructure and services described in this paper aims at sharing local grammars of linguistic phenomena (especially, MWUs) in the NLP community. It has the specificity of being decentralized: authors have their grammars on their own web 
site. An indexer is in charge of centralizing all the information on an access server. Users can freely publish, access and import grammars from it in a transparent manner. This library is presently limited to Unitex grammars, but we intend to extend it to other formats. We also plan to provide a corpus that have been annotated by the application of all grammars in order to show their coverage. The size of the library is rather small but it is slowly growing. We hope that it will encourage NLP researcher to share their grammars trough the system and then help new significant advances in the domain.

\section{References}

[1] R. Baeza-Yates and B. Ribeiro-Neto. 1999. Modern Information Retrival. Addison-Wesley.

[2] O. Blanc and M. Constant. 2006. Outilex, a platform for Text Processing. Proc. of ColingACL on Interactive Presentation Sessions. Sydney, Australia, pp. 73-76.

[3] O. Blanc, M. Constant and P. Watrin. 2007. Segmentation in super-chunks with a finite-state approach. Proc. of the Workshop on Finite State Methods for Natural Language Processing. Potsdam, Germany.

[4] Dolors Català and Jorge Baptista. 2007. Spanish Adverbial Frozen Expressions. Proc. of the Workshop on A Broader Perspective on Multiword Expressions. Prague, pp. 33-40.

[5] A. Copestake, F. Lambeau, A. Villavicencio, F. Bond, T. Baldwin, I. A. Sag and D. Flickinger. 2002. Multiword expressions: linguistic precision and reusability. Proc. of the Third conference on Language Resources and Evaluation. Las Palmas, Canary Islands, pp. 1941-1947.

[6] Nicole Grégoire 2007. Design and Implementation of a Lexicon of Dutch Multiword Expressions. Proc. of the Workshop on A Broader Perspective on Multiword Expressions. Prague, Czech Republic, pp. 17-24.

[7] Maurice Gross 1984. Lexicon-Grammar and the Syntactic Analysis of French. Proc. of the 10 th International Conference on Computational Linguistics. Stanford, California.

[8] Maurice Gross 1997. The construction of local grammars. In Roche, E., Schabes, Y. (eds.). Finite-State Language Processing. Cambridge, Mass., The MIT Press, pp. 329-352.

[9] J. Nam and K. Choi. 1997. A Local-Grammar-based Approach to Recognizing of Proper Names in Korean Texts. Zhou \& Church (eds.). Proc. of the Workshop on Very Large Corpora. ACL/Tsing-hua University/Hong-Kong University of Science and Technology, pp. 273-288.

[10] S. Paumier 2006. The Unitex Manual. http://igm.univ-mlv.fr/ unitex/.

[11] L. Page, S. Brin, R. Motwani and T. Winograd 1998. The PageRank Citation Ranking: Bringing Order to the Web. Stanford Digital Technologies.

[12] T. Qin, T.-Y. Liu, X.-D. Zhang, Z. Chen and W.-Y. MA 2005. A study of relevance propagation for web search Proc. of The 28th Annual International ACM SIGIR Conference. New York, NY : ACM Press.

[13] E. Ranchhod, P. Carvalho, C. Mota and A. Barreiro. 2004. Portuguese Large-scale Language Resources for NLP Applications. Proc. of Language Resources and Evaluation Conference. Lisbon, pp.1755-1758.

[14] Ivan A. Sag, Timothy Baldwin, Francis Bond, Ann A. Copestake and Dan Flickinger 2002. Multiword Expressions: A Pain in the Neck for NLP. Proc. of the Third International Conference on Computational Linguistics and Intelligent Text Processing, Springer-Verlag, London, UK, pp. 1-15.

[15] J. Senellart, M. Plitt, Ch. Bailly and F. Cardoso. 2001. Resource alignment and implicit transfer. Machine translation in the information age, MT Summit. pp. 317-323.

[16] M. Silberztein. 2003. Finite-state description of the french determiner system. Journal of French Language Studies. 13(2).

[17] W.A. Woods. 1970. Transition network grammars for natural language analysis. Communications of the ACM. 13(10). 